\title{
ON THE LITHIUM ABUNDANCE IN SUNSPOTS AND THE UNDISTURBED SOLAR ATMOSPHERE
}

\author{
E. Dubov, V. Prokof'ev, and A. Severny
}

The difference between abundances of such elements as $\mathrm{Li}, \mathrm{Be}, \mathrm{B}$ in active regions on the Sun and in the undisturbed atmosphere, if it exists at all, could be considered a very important indication on the possible nuclear reaction in active regions and on the rate of diffusion of elements inside magnetic fields of sunspots. One of the authors from the considerations of $\lambda 6707 \AA$ has an estimated (Dubov, 1955) $\mathrm{Li}$ abundance in the undisturbed solar atmosphere as $\log N(\mathrm{Li})=0.93$ (taking $\log N(H)$ $=12 \cdot 0$ ), which is close to the later results (Goldberg et al., 1960). In sunspots we can expect that the first doublet $\lambda 6103$ of $\mathrm{Li}$ diffuse series appears. The preliminary consideration (Dubov, 1964) showed that $\mathrm{Li}$ abundance in sunspots could be several times larger than the above estimate for the undisturbed atmosphere. This result forced us to consider as carefully as possible the region of $\lambda 6103$ in eight spectra

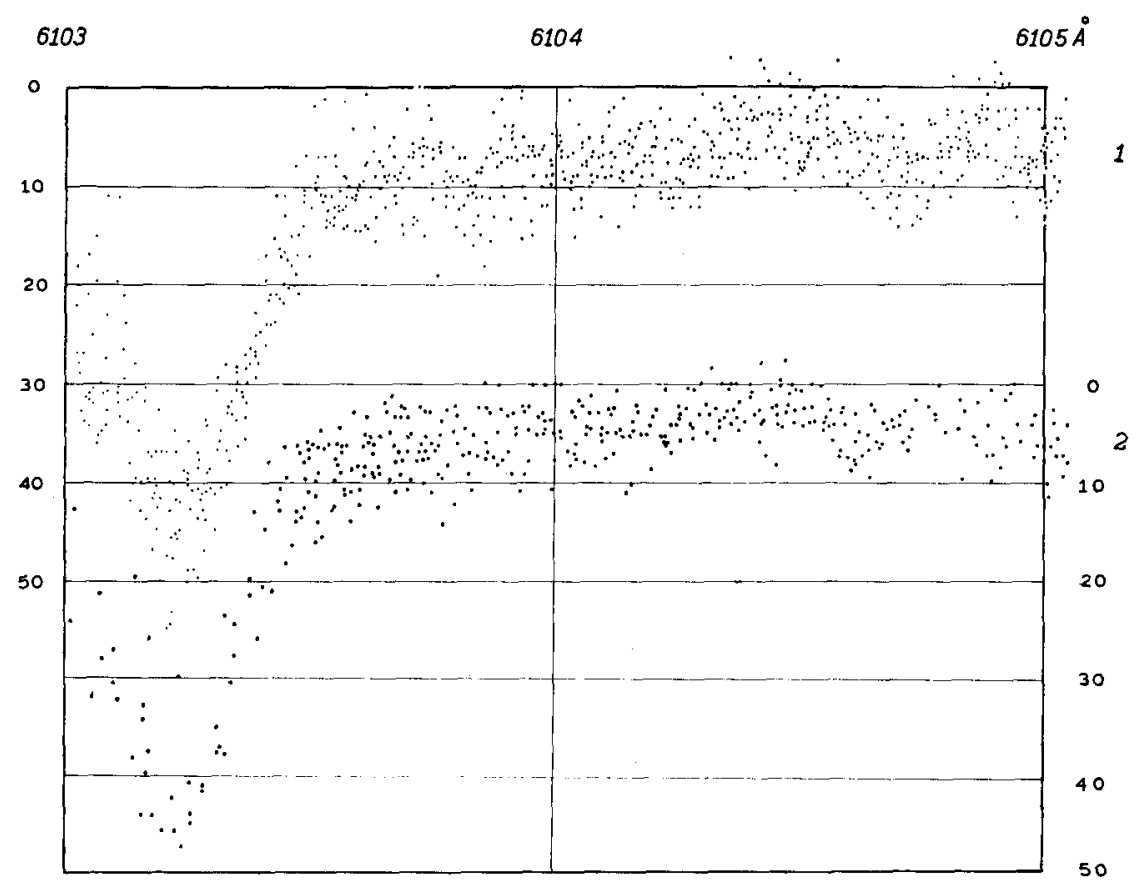

FIG. 1. Summary of all points of used spectra of different authors: $1=$ Prokofiev, $2=$ Severny.

Perek (ed.), Highlights of Astronomy, 247-250. O I.A.U. 


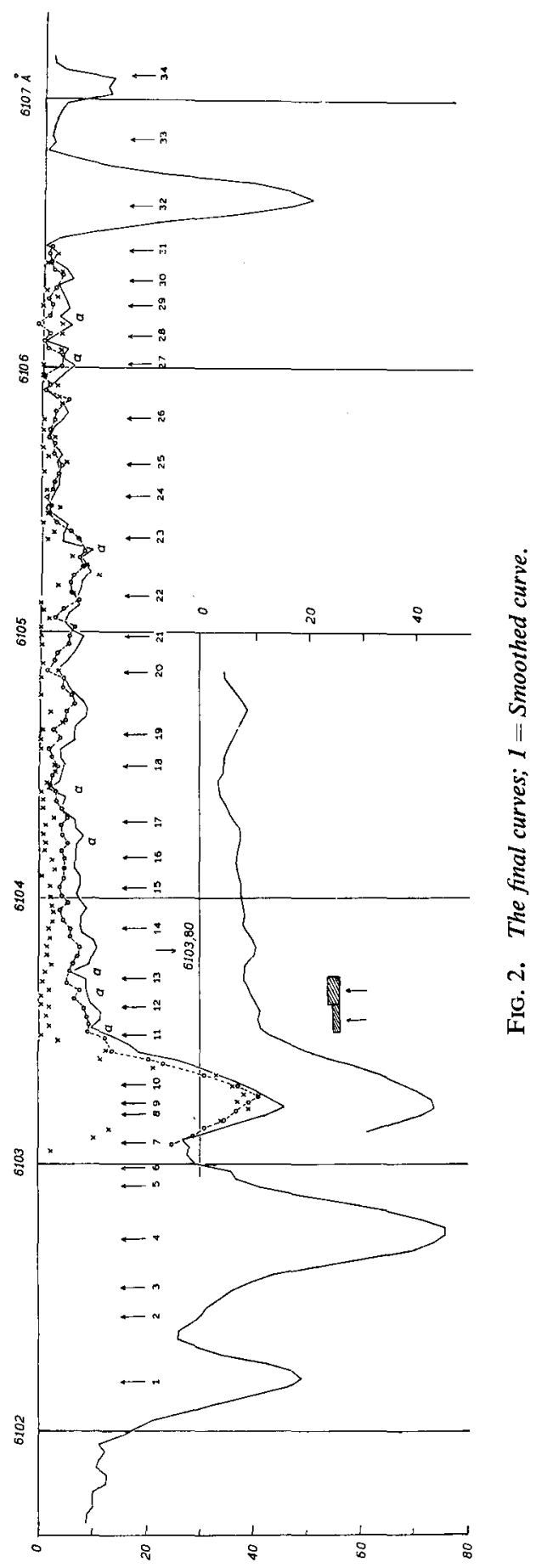


of four sunspots taken with the aid of the echelle-grating spectrograph of the Crimean observatory with a dispersion of $1 \AA / \mathrm{mm}$ and measured resolving power $0.03 \AA$. The consideration of the Zeeman pattern of this line shows that at the field strengths we had in sunspots (2000-3000 gs) the line should be diffuse band with width $\sim 0.23 \AA$, and taking into account the broadening of the line the halfwidth of the line has not to be smaller than $0.25 \AA$.

The careful spectrophotometry made by Prokofiev and Severny (1967) by two independent methods is shown in Figure 1 for all sunspot spectra we had at our disposal. In the same way the spectra of the undisturbed atmosphere were measured, and the mean profile of the region in question is shown in Figure 2 (crosses = undisturbed atmosphere; open circles = sunspot 1 st method; solid line = sunspot 2 nd method; arrows denote the positions of all lines known up to now in the spectrum of solar atmosphere; 12 corresponds to the line Fell $\lambda 6103.59)$. We see good agreement between different methods of reducing of spectrograms, and minor differences are connected with the different setting of the level of continuous spectrum. Figure 3 shows the same results obtained for different spots separately.

Careful inspection and analysis of these results shows that there is no indication, neither in the mean profile nor in the profile for separate sunspots, of the presence of some appreciable line in the place where we should expect the Li line $\lambda 6103.69$ (Kiasatpoor, 1965) (13 in Figures 2 and 3). The difficulty with the search of the Li line $\lambda 6103.65$ is increased by the influence of blends in this region (e.g. Fe II 6103.59) and due to some uncertainty with the position of Li line. If we adopt the laboratory system of wavelengths (the dashed rectangular areas in Figures 2 and 3), which is hardly adequate, the observed small depression in the region $\lambda 6103 \cdot 65$ (taking into

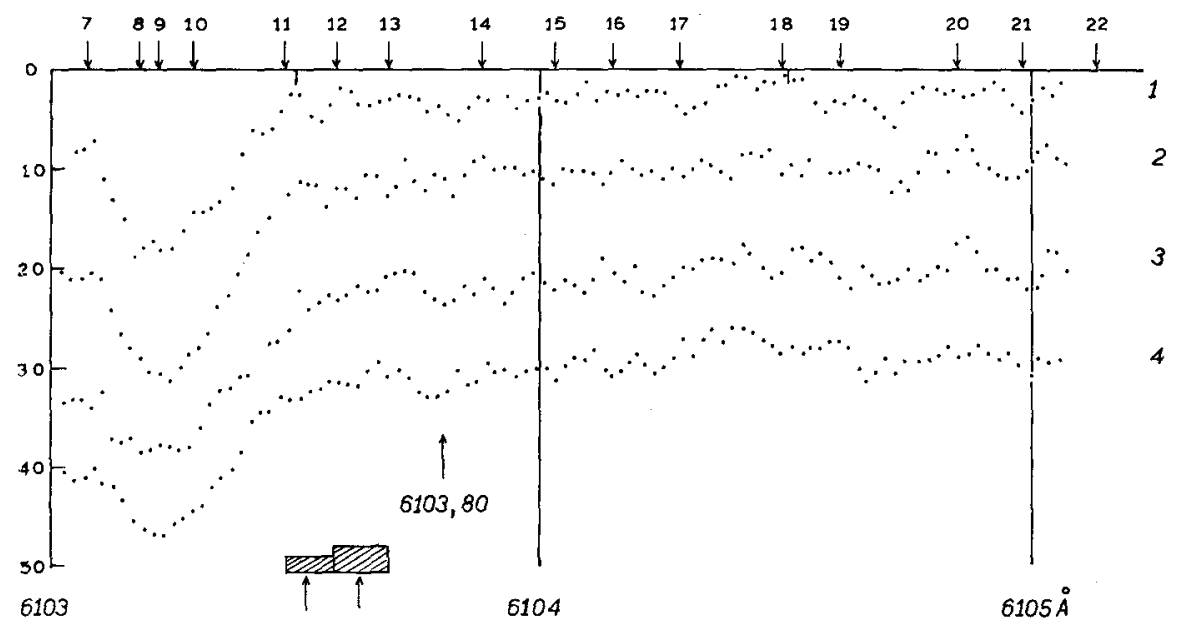

Fig. 3. The mean curves for different spots: $1=18.09 .1960 ; 2=2.09 .1961 ; 3=5.09 .1961$; $4=6.09 .1961$. 
account the influence of blend (FeII-line) would be less than $1.0 \mathrm{~m} \AA$; this is the mean for all eight spectra of four spots instead of $3.3 \pm 2.5 \mathrm{~m} \AA$ by Dubov and Hromova (1964) and found for one sunspot, which value includes possibly the influence of blends. This agrees with the estimate of the $\mathrm{Li}$ abundance by the same line $(1.1 \mathrm{~m} \AA)$ made by Schmahl and Schröter (1939) and the estimate made by Dubov (1967) from the consideration of the Li-resonance line $\lambda 6707$.

The abundances from equivalent widths have been derived by the method developed by Dubov and Hromova (1964).

Therefore at the present time we can hardly suspect some appreciable difference in Li abundance between undisturbed atmosphere and active regions on the Sun.

\section{References}

Dubov, E. (1955) Astron. Cirkular No. 159, p. 11.

Dubov, E. (1964) Izv. Krym. astrofiz. Obs., 32, 26.

Dubov, E. (1967) Izv. Krym. astrofiz. Obs., 36, 87.

Dubov, E., Hromova, T., (1964) Izv. Krym. astrofiz. Obs., 31, 247.

Goldberg, L., Müller, E.A., Aller, A.H. (1960) Astrophys. J., Suppl. 5, No. 45.

Kiasatpoor, A. (1965) Georgetown Obs., Monograph No. 19.

Prokofiev, V.K., Severny, A.B. (1967) Izv. Krym. astrofiz. Obs., 36, 90.

Schmahl, G., Schröter, E.H. (1939) Z. Astrophys., 18, 284. 